\title{
VARIÁVEIS BIOMÉTRICAS DA CANA-DE-AÇÚCAR FERTILIZADA COM RESÍDUOS ORGÂNICO E INDUSTRIAL E IRRIGADA COM ÁGUA SERVIDA E POTÁVEL ${ }^{1}$
}

\author{
FABIO O. DE NOBILE ${ }^{2}$, JOÃO A. GALBIATTI ${ }^{3}$, REGINALDO I. MURAISHI ${ }^{4}$, \\ JOSÉ R. ARAUJO
}

\begin{abstract}
RESUMO: O uso agrícola de resíduos orgânicos e industriais é uma alternativa de aproveitamento como fertilizante e fonte de matéria orgânica ao solo. Neste sentido, o objetivo deste trabalho foi avaliar o uso do composto de lixo e biofertilizante como fonte de nutrientes, e o resíduo do processamento da bauxita como corretivo da acidez do solo, para a cultura da cana-de-açúcar (cana-planta), irrigada com água potável e servida. Para tanto foi conduzido experimento em vaso, utilizando-se de solo Argissolo Vermelho-Amarelo distrófico, cultivado com a variedade RB855536. Foram avaliadas variáveis biométricas da planta, tais como: altura de plantas, diâmetro do colmo, perfilhamento, número de folhas, matéria seca de raiz e parte aérea. Os dados obtidos evidenciaram que a qualidade da água de irrigação, potável ou servida, não influenciou as variáveis avaliadas. A aplicação de composto de lixo e de biofertilizante constituiu-se numa fonte eficiente de nutrientes para as plantas de cana-de-açúcar, mas é necessária a aplicação adicional de fertilizantes para a obtenção de produção de colmos, similar ao do tratamento com adubação mineral convencional. Já o resíduo do processamento da bauxita mostrou-se eficiente na correção da acidez, apresentando as variáveis físicas semelhantes ao tratamento com adubação mineral, com exceção da matéria seca de raiz.
\end{abstract}

PALAVRAS-CHAVE: adubação orgânica, matéria seca, diâmetro de colmo, altura de plantas, raiz.

\section{BIOMETRIC VARIABLE OF SUGAR CANE FERTILIZED WITH ORGANIC AND INDUSTRIAL RESIDUES IRRIGATED WITH POTABLE AND WASTEWATER}

\begin{abstract}
The agricultural use of organic and industrial residues is an alternative of exploitation with fertilization and source of organic matter to the soil. In this direction, the objective of this research was to evaluate the use of the urban solid waste and biofertilizer as nutrient source, and residue of the bauxite processing as agricultural liming materials of the soil for sugar cane (cane plant) and culture yields, irrigated with potable water and wastewater. For in such an experiment in vase was lead, under Alfisol soil, cultivated with the variety RB855536. It had been evaluated biometric variable of the plant such as: plant height, diameter of stem, tillering, leaf number, dry matter of the root and dry matter of the aerial part. The data showed that the quality of the irrigation water, potable and wastewater, did not influence the evaluated parameters. The application of urban solid waste and biofertilizer had consisted in an efficient source of nutrients for sugar cane plants, but is necessary to apply additional fertilizer for the attainment of stem production similar to the treatment with conventional mineral fertilization. Now the residue of the bauxite processing revealed efficient in the correction of the acidity presenting similar physical parameters to the treatment with mineral fertilization and lime, with exception of the dry matter of the root.
\end{abstract}

KEYWORDS: organic fertilization, dry matter, diameter of stem, plant height, root.

\section{INTRODUÇÃO}

\footnotetext{
${ }^{1}$ Extraído da tese do primeiro autor e financiado pelo CNPq

${ }^{2}$ Prof. Doutor, Centro Universitário da Fundação Educacional de Barretos - SP, fonobile@ feb.br.

${ }^{3}$ Prof. Doutor, Titular do Departamento de Engenharia Rural, UNESP, Jaboticabal - SP, galbi@ fcav.unesp.br.

${ }^{4}$ Prof. M.Sc., Fundação Educacional de Barretos - SP, itimuraishi@ @otmail.com.

${ }^{5}$ Prof. M.Sc., Universidade Estadual de Minas Gerais, Frutal - MG, jrdedearaujo@uol.com.br.

Recebido pelo Conselho Editorial em: 8-12-2008
}

Aprovado pelo Conselho Editorial em: 10-1-2011 
A quantidade e a qualidade de água existente na natureza vêm diminuindo gradativamente, decorrente principalmente da urbanização, expansão agrícola, indústria e degradação do meio ambiente. Essas mudanças resultaram na escassez da água em várias regiões do mundo, dificultando o atendimento aos múltiplos usos a que se destina. Uma das alternativas para amenizar estes problemas, em muitas regiões, tem sido considerar o uso de águas de qualidade inferior para os mais variados setores da sociedade, como, por exemplo, a aplicação de água servida para a agricultura e indústria. O uso racional de água servida, considerando uma política definida, é um importante instrumento para minimizar a contaminação ambiental, as doenças de veiculação hídrica, que representam $65 \%$ das internações hospitalares no Brasil, como também podem melhorar a qualidade de vida da população (SOUSA et al., 2009).

A reutilização de resíduos é de grande interesse, pois além de dar destino aos mesmos, torna-os úteis, já que sua aplicação no solo interfere nos atributos químicos, aumentando a disponibilidade de nutrientes às plantas. Esses compostos contêm nutrientes, tais como N, P, K, Ca, $\mathrm{Mg}, \mathrm{B}, \mathrm{Cu}$, etc., que são elementos fundamentais para o desenvolvimento dos vegetais (FRANCO et al., 2010).

A utilização de compostos de lixo urbano e biofertilizante como condicionadores de solos agrícolas é uma prática em diversos países, como nos Estados Unidos, no Brasil, na Inglaterra, na Austrália e no Japão. Além do alto teor de matéria orgânica, há ainda a presença de nutrientes à planta, como o nitrogênio e o fósforo (NOBILE et al., 2006).

É importante salientar que um resíduo só deve ser incorporado ao solo quando não prejudicar suas propriedades e características originais, mas beneficiar de algum modo a adaptação das culturas. Vários tipos de rejeitos podem ser incorporados ao solo, tais como os resíduos de estações de tratamento de esgotos, desde que sejam controlados e monitorados. Os riscos da disposição indiscriminada de resíduos no solo associam-se principalmente ao transporte de poluentes pela infiltração de líquidos percolados, responsáveis pela contaminação do subsolo e das águas subterrâneas nos locais de disposição.

O resíduo do refino de bauxita pode ser uma opção como corretivo de solo, uma vez que possui elevado $\mathrm{pH}$ e grande quantidade de hidroxilas (grupamentos $\mathrm{OH}$ ) oriundos da adição de $\mathrm{NaOH}$ para extração de alumínio; por outro lado, tem como excesso o sódio $(\mathrm{Na})$, que pode ser um agravante no seu uso na agricultura.

O objetivo deste trabalho foi avaliar o uso do composto de lixo e biofertilizante como fonte de nutrientes, e o resíduo do processamento da bauxita como corretivo da acidez do solo, para a cultura da cana-de-açúcar (cana-planta), irrigada com água potável e servida.

\section{MATERIAL E MÉTODOS}

O experimento foi conduzido em ambiente protegido do tipo arco conjugado, coberto por filme plástico de polietileno e tela antiafídica em toda sua área externa, localizado no setor de Plasticultura do Departamento de Engenharia Rural, da Faculdade de Ciências Agrárias e Veterinárias - Câmpus de Jaboticabal - SP, cujas coordenadas geográficas são $21^{\circ} 15^{\prime} 15^{\prime}$ ' latitude sul, 48¹8'09' longitude oeste e altitude em torno de $595 \mathrm{~m}$. Utilizou-se solo oriundo do Argissolo Vermelho-Amarelo distrófico, arênico, retirado na camada de 0-20 cm, cujas propriedades químicas são: $\mathrm{pH}_{\mathrm{CaCl} 2}=4,7$; M.O. $\left(\mathrm{g} \mathrm{dm}^{-3}\right)=7 ; \mathrm{P}\left(\mathrm{mg} \mathrm{dm}^{-3}\right)=8 ; \mathrm{K}, \mathrm{Ca}, \mathrm{Mg}, \mathrm{H}+\mathrm{Al}, \mathrm{SB}, \mathrm{T}$ e Al $\left(\mathrm{mmol}_{\mathrm{c}} \mathrm{dm}^{-3}\right)$ $=1,1 ; 6 ; 4 ; 16 ; 11,1 ; 27,1 \mathrm{e} 1$, respectivamente, $\mathrm{V}(\%)=41$. As análises químicas foram realizadas conforme metodologia de RAIJ et al. (2001), sendo a densidade do solo de $1,25 \mathrm{~kg} \mathrm{dm}^{-3}$.

A fonte de água, considerada como "água servida", foi coletada no Córrego Jaboticabal, onde é despejada parte do esgoto residencial, produzido pela cidade de Jaboticabal - SP. A "água potável" era proveniente de poço artesiano do reservatório central do Câmpus, que abastece as edificações do setor de Plasticultura, UNESP/FCAV (Tabela 1). 
TABELA 1. Dados da análise química das águas de irrigação. Data of the chemical analysis of irrigation waters.

\begin{tabular}{|c|c|c|c|c|c|c|c|c|}
\hline \multirow{2}{*}{ água } & C.E. & $\mathrm{pH}$ & $\mathrm{N}-\mathrm{NH}_{3}$ & $\mathrm{PO}_{4}$ & $\mathrm{~K}$ & $\mathrm{Ca}$ & $\mathrm{Mg}$ & $\mathrm{S}-\mathrm{SO}_{4}$ \\
\hline & $\mathrm{dS} \mathrm{m}^{-1}$ & $\mathrm{CaCl}_{2}$ & \multicolumn{6}{|c|}{ - } \\
\hline Servida & 0,82 & 6,9 & 0,87 & 0,705 & 0 & 0,05 & 0 & 0,01 \\
\hline Potável & 0,63 & 6,3 & 0,29 & 0,353 & 0 & 0,06 & 0 & 0,01 \\
\hline
\end{tabular}

Metodologia utilizada: APHA (2005); C.E. - condutividade elétrica.

O resíduo do processamento da bauxita, em condições de ser empregado como condicionador de solo, foi obtido junto à empresa Alcoa Alumínio S.A. (MG). O composto de lixo urbano foi obtido na cidade de São José do Rio Preto - SP, junto à empresa Constroeste Ambiental. É resultante do aproveitamento racional de lixo doméstico urbano. O biofertilizante utilizado foi obtido junto ao Departamento de Engenharia Rural, da Faculdade de Ciências Agrárias e Veterinárias - Câmpus de Jaboticabal - SP, sendo gerado na digestão anaeróbia de dejetos de suíno, obtido através da limpeza das instalações desses animais em criação intensiva, nas fases de crescimento e terminação, cujas análises se apresentam na Tabela 2.

TABELA 2. Dados da análise química dos resíduos orgânicos e industrial, base seca. Data of the chemical analysis of the organic and industrial residues, dry basis.

\begin{tabular}{lccccccccccc}
\hline & $\mathrm{N}$ & $\mathrm{P}$ & $\mathrm{K}$ & $\mathrm{Ca}$ & $\mathrm{Mg}$ & $\mathrm{S}$ & $\mathrm{Fe}$ & $\mathrm{Mn}$ & $\mathrm{Cu}$ & $\mathrm{Zn}$ & $\mathrm{Na}$ \\
\cline { 2 - 11 } & $--------------\mathrm{g} \mathrm{kg}^{-1}$ & -------------- & -------------- & $\mathrm{mg} \mathrm{kg}^{-1}$ & -------------- \\
\hline Res. proc. da bauxita & 4 & 0,001 & 11,4 & 17 & 1 & 0,8 & 137.000 & 2.100 & 4 & 111 & 50.100 \\
Composto de lixo & 15 & 4 & 6 & 31 & 4 & 4,2 & 25.300 & 350 & 67 & 1.100 & 2.650 \\
Biofertilizante & 140 & 54 & 48 & 65 & 26 & 20 & 1 & 0,20 & 0,1 & 1,4 & 18 \\
\hline
\end{tabular}

Metodologia utilizada: LANARV (1988)

Para a instalação do experimento, foram construídos recipientes de PVC com dimensões de $45 \mathrm{~cm}$ de altura e $30 \mathrm{~cm}$ de diâmetro, totalizando um volume de $32 \mathrm{~L}$. Os tratamentos testados resultaram em cinco tipos de fertilização: a) sem adubação; b) fertilização mineral; c) fertilização com resíduo do processamento da bauxita; d) fertilização com composto de lixo urbano; e) fertilização com biofertilizante oriundo da digestão anaeróbia de dejetos suínos e dois tipos de irrigação (água potável e água servida residencial), totalizando 10 tratamentos. Assim, o delineamento experimental foi o em blocos casualizados, em esquema fatorial $5 \times 2$, com três repetições, totalizando 30 parcelas. Os 10 tratamentos resultantes das combinações mencionadas foram agrupados em três blocos, sendo cada parcela experimental constituída de um vaso com uma planta de cana-de-açúcar.

O solo foi peneirado em peneira de malha com $20 \mathrm{~mm}$, procurando manter a integridade dos torrões até a abertura da malha, para retirar todos os agregados maiores e resíduos grosseiros de material orgânico. O volume de solo da camada de 0-20 cm foi calculado em função do raio do recipiente $(30 \mathrm{~cm})$ e da altura da camada de solo $(20 \mathrm{~cm})$, totalizando um volume de $14,14 \mathrm{dm}^{-3}$. A quantidade de solo para essa camada, foi calculada em função da densidade do solo $\left(1,25 \mathrm{~kg} \mathrm{dm}^{-3}\right) \mathrm{e}$ volume do recipiente $\left(14,14 \mathrm{dm}^{-3}\right)$, totalizando $17,68 \mathrm{~kg}$ de solo.

Depois de calculada a quantidade de solo da camada de 0-20 cm foram feitas aplicações de calcário e resíduo do processamento da bauxita para os respectivos tratamentos, com o objetivo de diminuir a acidez do solo. As quantidades de resíduo do processamento de bauxita e calcário foram calculadas através do método de saturação por bases. Após a aplicação, o solo passou por um período de 90 dias de incubação.

Finalizado o período de incubação, o solo tratado foi novamente seco e peneirado em peneira de malha $20 \mathrm{~mm}$, sendo a partir daí realizada a adição dos resíduos orgânicos e dos fertilizantes 
minerais, com base na análise química do solo e de acordo com as recomendações de RAIJ et al. (1997).

Na Tabela 3, são apresentadas as quantidades de fertilizantes, corretivos e resíduos utilizados nos tratamentos.

TABELA 3. Quantidades de fertilizantes, corretivos e resíduos usados nos tratamentos. Amounts of fertilizers, correctives and residues used in the treatments.

\begin{tabular}{|c|c|c|c|}
\hline \multirow[b]{2}{*}{ Tratamento } & \multirow{2}{*}{ Fertilizantes, corretivos e resíduos } & \multicolumn{2}{|c|}{ Quantidades } \\
\hline & & $\mathrm{kg} \mathrm{ha}^{-1}$ & g vaso $^{-1}$ \\
\hline Testemunha & --- & --- & --- \\
\hline \multirow{4}{*}{ Fertilização Mineral } & - calcário $(\mathrm{MgO}=9 \%$ e PRNT=95\%) & 600 & 4,25 \\
\hline & - nitrato de cálcio & 195 & 1,40 \\
\hline & - superfosfato simples & 800 & 5,70 \\
\hline & - cloreto de potássio & 230 & 1,60 \\
\hline \multirow{4}{*}{ Res. do proc. de bauxita } & - nitrato de cálcio & 195 & 1,40 \\
\hline & - superfosfato simples & 800 & 5,70 \\
\hline & - cloreto de potássio & 230 & 1,60 \\
\hline & -res. do proc. de bauxita & 4.300 & 30,33 \\
\hline \multirow{2}{*}{ Composto de lixo } & - calcário $(\mathrm{MgO}=9 \%$ e PRNT=95\%) & 600 & 4,25 \\
\hline & - composto de lixo & 20.000 & 141,38 \\
\hline \multirow{2}{*}{ Biofertilizante } & - calcário $(\mathrm{MgO}=9 \%$ e PRNT=95\%) & 600 & 4,25 \\
\hline & biofertilizante & 40.000 & 282,75 \\
\hline
\end{tabular}

Com o auxílio de sacos de plástico, o solo foi misturado $(17,68 \mathrm{~kg})$ com resíduos orgânicos (composto de lixo e biofertilizante) e fertilizantes. Após a mistura do solo com os tratamentos estabelecidos e antes do acondicionamento nos recipientes, foi feita adição de solo preenchendo a camada de 20-45 cm. O volume de solo para essa camada foi calculado em função do raio do recipiente $(30 \mathrm{~cm})$ e da altura $(25 \mathrm{~cm})$, totalizando um volume de $17,68 \mathrm{dm}^{-3}$. Com o volume do vaso e a densidade do solo, calculou-se a quantidade de solo necessária para o preenchimento da camada em questão $(22,11 \mathrm{~kg})$. Após o preenchimento dos primeiros $25 \mathrm{~cm}$, foi adicionado o restante do solo, preenchendo os outros $20 \mathrm{~cm}$, totalizando $45 \mathrm{~cm}$ de altura do recipiente.

Depois do preenchimento do recipiente, procedeu-se ao transplantio da cana-de-açúcar, variedade RB855536, em 30 de agosto de 2006. Foram utilizadas mudas provenientes de cultura de tecidos, apresentando uma planta de cana-de-açúcar por muda.

Após 50 dias do plantio da cana-de-açúcar, foi feita a adubação de cobertura, aplicando $50 \mathrm{~kg} \mathrm{ha}^{-1}$ de nitrogênio e $20 \mathrm{~kg} \mathrm{ha}^{-1}$ de potássio, ou seja, 3,25 gramas de nitrato de cálcio e $0,30 \mathrm{~g}$ de cloreto de potássio. Essa adubação foi feita apenas para os tratamentos de fertilização, com resíduo do processamento da bauxita e fertilização mineral. A partir da instalação do experimento, foram feitas irrigações diárias de acordo com a evapotranspiração. A colheita do experimento foi realizada no dia 23 de abril de 2007.

Na irrigação, foi utilizado atmômetro modificado que pode estimar a evapotranspiração de referência com boa precisão, cujas medidas de evaporação foram tomadas com base para definir as lâminas de água a serem aplicadas na irrigação. O Kc da cultura da cana-de-açúcar, utilizado para o cálculo da quantidade de água a ser aplicada por vaso, seguiu a metodologia sugerida por HERNANDEZ (2008), em que o Kc inicial foi 0,4; fase de desenvolvimento $\mathrm{Kc}=0,7$; período intermediário $\mathrm{Kc}=1,0$; final do ciclo $\mathrm{Kc}=0,75$; colheita $\mathrm{Kc}=0,5$. Assim, a evapotranspiração da cultura é obtida multiplicando-se a evapotranspiração de referência pelo coeficiente de cultura (Kc) [(eq.(1)]: 
Antes da colheita do experimento, foi feita medição de altura de plantas (medida a partir da superfície do solo até a extremidade da folha mais alta com colarinho visível, medindo-se duas plantas por parcela. A medição foi feita com trena graduada (em centímetros), número de folhas (foi feita a contagem do número de folhas por planta existentes em cada vaso), diâmetro do colmo (foi usado paquímetro digital, avaliando medidas do centro dos colmos da planta, a uma altura de $60 \mathrm{~cm}$ do solo e escolhendo-se duas plantas por parcela) e número de plantas (feito através da contagem manual do número de plantas existentes em cada vaso). A cana-de-açúcar foi cortada rente ao solo e, logo em seguida, levada à estufa para a secagem, sendo posteriormente pesada para a obtenção da matéria seca da parte aérea por recipiente, em $\mathrm{g} \mathrm{vaso}^{-1}$. A matéria seca da raiz foi obtida após a retirada do material dos recipientes, lavagem para retirada de solo e secagem em estufa, obtendo a matéria seca de raiz, em $\mathrm{g}$ vaso $^{-1}$.

\section{RESULTADOS E DISCUSSÃO}

Os maiores diâmetros de colmos foram observados para os tratamentos que receberam fertilizantes e corretivos, ou seja, os tratamentos com adubação mineral e com resíduo do processamento da bauxita, respectivamente, com 21,11 e $21,21 \mathrm{~cm}$, seguido pelos tratamentos com composto de lixo e com biofertilizante, apresentando diâmetro de colmo de 19,74 e $20,75 \mathrm{~cm}$. O menor diâmetro foi observado para o tratamento-testemunha, com 17,03 cm (Tabela 4). Não foi observado efeito da qualidade da água de irrigação, potável ou servida, no desenvolvimento dos colmos de cana-de-açúcar.

NOBILE et al. (2010), estudando a aplicação de resíduo do processamento da bauxita, observou que o maior diâmetro de colmo ocorreu com a aplicação de $28 \mathrm{t} \mathrm{ha}^{-1}$. Já MATHEUS (2004) encontrou aumento no diâmetro de colmos de milho com a combinação de $2 \mathrm{t} \mathrm{ha}^{-1}$ de biofertilizante mais metade da recomendação de fertilizantes minerais, obtendo diâmetros de $22 \%$.

TABELA 4. Dados da análise biométrica da planta de cana-de-açúcar. Data of the biometric analysis of sugar cane.

\begin{tabular}{lcccccc}
\hline Tratamentos (T) & $\begin{array}{c}\text { Diâmetro do } \\
\text { Colmo } \\
\text {----- cm ----- }\end{array}$ & $\begin{array}{c}\text { Altura de } \\
\text { Plantas }\end{array}$ & $\begin{array}{c}\text { Número de } \\
\text { Folhas }\end{array}$ & $\begin{array}{c}\text { Número de } \\
\text { Perfilhos }\end{array}$ & $\begin{array}{c}\text { Matéria Seca } \\
\text { de Raiz }\end{array}$ & $\begin{array}{c}\text { Matéria Seca } \\
\text { da Parte Aérea }\end{array}$ \\
\hline Testemunha & $17,03 \mathrm{~d}$ & $2,48 \mathrm{~b}$ & $5,00 \mathrm{c}$ & $1,83 \mathrm{~b}$ & $61,66 \mathrm{~d}$ & $585,00 \mathrm{c}$ \\
Ad. mineral & $21,11 \mathrm{a}$ & $2,79 \mathrm{a}$ & $10,00 \mathrm{a}$ & $3,83 \mathrm{a}$ & $115,47 \mathrm{a}$ & $763,33 \mathrm{a}$ \\
Res. Proc. bauxita & $21,21 \mathrm{a}$ & $2,74 \mathrm{a}$ & $9,00 \mathrm{a}$ & $3,66 \mathrm{a}$ & $80,91 \mathrm{c}$ & $749,93 \mathrm{a}$ \\
Composto de lixo & $19,74 \mathrm{~b}$ & $2,46 \mathrm{~b}$ & $7,50 \mathrm{~b}$ & $1,50 \mathrm{~b}$ & $82,75 \mathrm{c}$ & $718,33 \mathrm{~b}$ \\
Biofertilizante & $20,15 \mathrm{~b}$ & $2,54 \mathrm{~b}$ & $7,00 \mathrm{~b}$ & $3,00 \mathrm{a}$ & $105,09 \mathrm{~b}$ & $725,00 \mathrm{~b}$ \\
\hline Teste (F) & $157,05^{* *}$ & $54,06^{* *}$ & $4,45^{* *}$ & $13,91^{* *}$ & $471,37^{* *}$ & $92,54^{* *}$ \\
DMS & 0,67 & 0,09 & 1,07 & 1,08 & 4,32 & 24,80 \\
\hline \hline Água (A) & & & & & \\
\hline Potável (1) & 21,00 & 2,65 & 7,26 & 2,80 & 89,64 & 726,42 \\
Servida (2) & 20,83 & 2,62 & 7,73 & 2,33 & 88,51 & 735,33 \\
\hline Teste (F) & $2,32^{\mathrm{NS}}$ & $3,58^{\mathrm{NS}}$ & $2,36^{\mathrm{NS}}$ & $4,36^{\mathrm{NS}}$ & $3,666^{\mathrm{NS}}$ & $1,92^{\mathrm{NS}}$ \\
DMS & 0,29 & 0,04 & 0,47 & 0,47 & 1,89 & 13,91 \\
T x A & $0,61^{\mathrm{NS}}$ & $0,65^{\mathrm{NS}}$ & $0,36^{\mathrm{NS}}$ & $1,02^{\mathrm{NS}}$ & $0,65^{\mathrm{NS}}$ & $0,33^{\mathrm{NS}}$ \\
C.V. (\%) & 1,95 & 1,96 & 8,07 & 13,85 & 2,79 & 2,53 \\
\hline
\end{tabular}

${ }^{\mathrm{ns}}$ não significativo. * $\mathrm{e}^{* *}$ significativo a 5 e a $1 \%$ de probabilidade pelo teste $\mathrm{F}$, respectivamente.

As maiores alturas de plantas foram verificadas para os tratamentos com adubação mineral e com resíduo do processamento da bauxita, respectivamente, com 2,79 e 2,74 m. Os tratamentos compostos de lixo e biofertilizante apresentaram alturas, respectivamente, de 2,46 e 2,54 m, que foram semelhantes, estatisticamente, ao tratamento-testemunha $(2,48 \mathrm{~m})$. Cabe ressaltar que os tratamentos adubação mineral e resíduo do processamento da bauxita novamente foram superiores devido ao fornecimento em quantidades adequadas e proporções convenientes de fertilizantes 
minerais. A qualidade de água não influenciou na altura de plantas, ou seja, o uso de água potável ou água servida na irrigação produziu os mesmos efeitos.

VILLELA JÚNIOR et al. (2003) observaram maior desenvolvimento de plantas de melão, em sistema hidropônico, com o uso de $100 \%$ de solução nutritiva quando comparado ao uso de biofertilizante. NOBILE et al. (2005) verificaram aumento de altura de plantas de $11 \%$ com a aplicação de $56 \mathrm{t} \mathrm{ha}^{-1}$ de resíduo do processamento de bauxita. GALBIATTI et al. (2007) observaram maior altura de plantas para o tratamento com a aplicação de composto de lixo na proporção de $1,00 \mathrm{dm}^{3}$ de composto para $1,00 \mathrm{dm}^{3}$ de solo.

Todos os tratamentos apresentaram número médio de folhas superior, estatisticamente, ao tratamento-testemunha, sendo os maiores números de folhas observados para os tratamentos com adubação mineral e com resíduo do processamento da bauxita, respectivamente, com 10,00 e 9,00 folhas por planta. Já os tratamentos com composto de lixo e com bioferilizante, que foram iguais estatisticamente, mas inferiores aos tratamentos com adubação mineral e com resíduo do processamento da bauxita, apresentaram 7,50 e 7,00 folhas por planta, respectivamente. Tratamentos que receberam algum tipo de fertilizante e/ou corretivo apresentaram respostas, neste caso, no aumento do número de folhas. O tipo de água usado na irrigação não mostrou diferenças estatísticas em relação ao número de folhas de cana-de-açúcar.

$\mathrm{Na}$ Tabela 4, pode-se verificar que os tratamentos com adubação mineral, com resíduo do processamento da bauxita e com biofertilizante, apresentaram maiores perfilhamentos, respectivamente, com 3,83; 3,66 e 3,00 perfilhos por vaso. Já os tratamentos-testemunha e composto de lixo não apresentaram diferenças estatísticas, sendo os menores perfilhamentos, respectivamente, com 1,83 e 1,50 perfilho por vaso.

Para FRANCO et al. (2010), dentre os elementos minerais de plantas, os mais importantes para o perfilhamento são o nitrogênio e o fósforo. NOBILE et al. (2010) não observaram influência do resíduo do processamento da bauxita nas diferentes doses testadas sobre o perfilhamento da cana-de-açúcar. ALBUQUERQUE et al. (2010) observaram efeito positivo na aplicação do composto de lixo no perfilhamento, quando comparado ao uso de fertilizantes químicos, constatando aumentos de $85 \%$ no perfilhamento.

Os dados apresentados na Tabela 4 mostraram que todos os tratamentos, com exceção dos tratamentos com composto de lixo e resíduo do processamento da bauxita, diferiram entre si em relação ao peso de matéria seca da raiz, sendo que o maior peso foi observado para tratamento com adubação mineral $\left(115,47 \mathrm{~g}\right.$ vaso $\left.^{-1}\right)$. A fertilização com resíduo do processamento da bauxita apresentou massa inferior ao tratamento adubação mineral, de acordo com KIEHL (1985), citado por NOBILE et al. (2010); o uso de resíduo com alta concentração de sódio provoca aumento do potencial osmótico da solução do solo, diminuindo sua disponibilidade de água e levando à diminuição do crescimento radicular.

Outra possibilidade, citada por NOBILE et al. (2007), para a diminuição radicular do tratamento com resíduo do processamento da bauxita, está no efeito desagregante do sódio no solo, favorecendo a compactação e dificultando a penetração das raízes.

O tratamento com biofertilizante apresentou a segunda maior matéria seca de raízes $\left(105,09 \mathrm{~g} \mathrm{vaso}^{-1}\right)$. Esse efeito é devido à grande quantidade de matéria orgânica adicionada ao solo, que, segundo KIEHL (1985) citado por NOBILE et al. (2010), tem como principal função o aumento radicular, devido às melhorias nas propriedades físicas, químicas e biológicas do solo. A menor matéria seca foi verificada para o tratamento testemunha $\left(61,66 \mathrm{~g} \mathrm{vaso}^{-1}\right)$, pois o mesmo não recebeu nenhum tipo de fertilizante e corretivo, tendo assim menores condições para $o$ desenvolvimento radicular. De acordo com a Tabela 4, a qualidade da água (potável ou servida) não interferiu estatisticamente na matéria seca da raiz.

Cabe ressaltar que a aplicação do resíduo do processamento de bauxita apresentou as maiores médias biométricas para cana-de-açúcar, entretanto a matéria seca de raiz obteve o menor peso 
(80,91 $\left.\mathrm{g} \mathrm{vaso}^{-1}\right)$, com exceção do tratamento-testemunha, deixando claro que o excesso de sódio é um agravante e que aplicações sucessivas do subproduto podem afetar negativamente $o$ desenvolvimento de plantas.

Todos os tratamentos apresentaram aumentos significativos, diferindo estatisticamente do tratamento-testemunha, sendo que a maior matéria seca da parte aérea foi verificada para os tratamentos com adubação mineral e com resíduo do processamento da bauxita, respectivamente, com 763,33 e 749,93 $\mathrm{g}_{\text {vaso }}{ }^{-1}$, seguido pelos tratamentos com composto de lixo e biofertilizante, respectivamente, com 718,33 e 725,00 $\mathrm{g} \mathrm{vaso}^{-1}$ (Tabela 4).

Os resultados mostraram que aplicações de quantidades adequadas de fertilizantes e corretivos promovem os melhores desempenhos da cultura, já que o uso de composto de lixo e biofertilizante, mesmo tendo recebido calcário para correção da acidez do solo, não tem capacidade de fornecer todos os nutrientes em quantidades adequadas e suficientes para a planta, necessitando de complementação. STAMFORD et al. (2006), trabalhando com biofertilizante, também encontraram efeito na matéria seca dos colmos da cana-de-açúcar, cultivada em solo de tabuleiro costeiro da Zona da Mata de Pernambuco, sendo os resultados semelhantes aos encontrados no presente trabalho.

\section{CONCLUSÕES}

A qualidade da água de irrigação não afetou nenhuma variável relacionada à planta.

Todos os tratamentos aumentaram a produção de matéria seca da raiz e da parte aérea, diâmetro de colmos e o número de folhas, em relação à testemunha; a altura de plantas só aumentou com o uso de adubação mineral e resíduo do processamento da bauxita; o perfilhamento apenas aumentou com a aplicação de adubos minerais, resíduo do processamento da bauxita e biofertilizante.

\section{AGRADECIMENTO}

Ao CNPq, pelo auxílio concedido durante a execução do projeto.

\section{REFERÊNCIAS}

ALBUQUERQUE, A.W.; ROCHA, E.S.; COSTAI, J.P.V. da; FARIAS, A.P.; BASTOS, A.L. Produção de helicônia Golden Torch influenciada pela adubação mineral e orgânica. Revista Brasileira de Engenharia Agrícola e Ambiental, Campina Grande, v.14, n.10, p.1.052-1.058, 2010.

APHA. AMERICAN PUBLIC HEALTH ASSOCIATION. Standart methods for the examination of water and wastewater. $21^{\text {th }}$ ed. Washington, 2005. $1.220 \mathrm{p}$.

FRANCO, H.C.J.; TRIVELINI; P.C.O.; FARONI, C.E.; VITTI, A.C.; OTTO, R. Stalk yield and technological attributes of planted cane as related to nitrogen fertilization. Scientia Agricola, Piracicaba, v.67, n.5, p.579-590, 2010 .

GALBIATTI, J.A.; LUI, J.J.; SABONARO, D.Z.; BUENO, L.F.; SILVA, V.L. da. Formação de mudas de eucalipto com utilização de lixo orgânico e níveis de irrigação calculados por dois métodos. Engenharia. Agrícola, Jaboticabal, v.27, n.2, p.445-455, 2007 .

HERNANDEZ, F.B.T. Manejo da Irrigação, abril 2008. Disponível em: <http://www.agr.feis.unesp.br/curso3.htm>. Acesso em: 30 out. 2009.

KIEHL, J.E. Fertilizantes orgânicos. Piracicaba: Agronômica Ceres, 1985. 492 p.

LABORATÓRIO NACIONAL DE REFERÊNCIA VEGETAL - LANARV. Análise de corretivos, fertilizantes e inoculantes: métodos oficiais do Laboratório Nacional de Referência Vegetal.

Brasília: LANARV, 1988. 104 p. 
MATHEUS, J. E. Evaluación agronómica del uso de compost de residuos de la industria azucarera (biofertilizante) en el cultivo de maíz (Zea mays). Bioagro, Trujillo, v.16, n.3, p.219-224, 2004.

NOBILE, F.O. de; GALBIATTI, J.A.; ANDRIÃO, M.A.; RIBEIRO, A.G.; MURAISHI, R.I. Quantificação da biomassa de cana-de-açúcar em substrato de resíduo de bauxita. Arquivos do Instituto Biológico, São Paulo, v.72, p.53, 2005.

NOBILE, F.O. de; GALBIATTI, J.A.; CORDIDO, J.P.B.R.; ANDRIÃO, M.A.; MURAISHI, R.I. Matéria orgânica e $\mathrm{pH}$ em solo adubado com fertilizantes orgânicos e minerais e irrigada com água residuária. In: REUNIÃO BRASILEIRA DE MANEJO E CONSERVAÇÃO DO SOLO E DA ÁGUA, 16., 2006, Aracaju. Resumos... Aracaju: SBCS, 2006. 1 CD-ROM.

NOBILE, F.O. de; GALBIATTI, J.A.; MURAISHI, R.I.; CORDIDO, J.P.B.R.; ANDRIÃO, M.A. Doses de composto de lixo no substrato e dois níveis de irrigação em crisântemo. In: CONGRESSO BRASILEIRO DE ENGENHARIA AGRÍCOLA, 36., 2007, Bonito. Anais... Bonito: SBEA, 2007. 1 CD-ROM.

NOBILE, F.O. de; GALBIATTI, J.A.; MURAISHI, R.I.; RIBEIRO, A.G.; FREDDI, O.S. Quantificação de macronutrientes no solo e em folhas de cana-de-açúcar em função de doses de resíduo da mineração de bauxita. Engenharia Agrícola, Jaboticabal, v.30, n.1, p.168-178, 2010 .

RAIJ, B. van; ANDRADE, J.C. de; CANTARELLA, H.; QUAGGIO, J.A. Análise química para avaliação da fertilidade de solos tropicais. Campinas: Instituto Agronômico, 2001. 285 p.

RAIJ, B. van; CANTARELLA, H.; QUAGGIO, J.A.; FURLANI, A.M.C. (Eds.) Recomendações de adubação e calagem para o Estado de São Paulo. 2.ed. Campinas: Instituto Agronômico de Campinas, 1997. p.39. (Boletim Técnico, 100).

SOUSA, J.T.; ARAÚJO, H.C.; CATUNDA, P.F.C. Reúso de esgotos sanitários para a agricultura. Disponível em: <http://www.iica.org.br/AguaTrab/Jose\%20Tavares/P2TB06.html. Fev. de 2009> Acesso em: 2 jun. 2009.

STAMFORD, N. P.; LIMA, R. A.; SANTOS, C. E. R.S.; DIAS, S. H. L. Biofertilizers with Acidithiobacillus on sugarcane yield and nutrients uptake in a tableland soil. Geomicrobiology, London, v.23, p.261-265, 2006.

VILLELA JÚNIOR, L.V.E.; ARAÚJO, J.A.C. de; FACTOR, T. Estudo da utilização do efluente de bioditgestor no cultivo hidropônico do meloeiro. Revista Brasileira de Engenharia Agrícola e Ambiental, Campina Grande, v.7, n.1, p.72-79, 2003. 